\title{
Dialogue based recommender system that flexibly mixes utterances and recommendations
}

\author{
Daisuke Tsumita \\ Dept. of Computer Science, \\ Meiji University, Japan \\ tsumidai@cs.meiji.ac.jp
}

\begin{abstract}
Much of the prior research in the recommendations through dialogue separate dialogue and recommendations. However, since the accuracy of the recommendations themselves is not necessarily high, recommendation results rarely meet user needs. However, as human we can find the solutions that satisfy users by appropriately repeating the cycle of checking mismatched reasons and making another recommendations in our conversations. In this paper, we propose a system for leveraging a dialogue strategy for reinforcement learning using recommendation results based on user utterances. We constructed a dialogue system to perform adaptive behavior that naturally incorporates recommendations into conversation with users.
\end{abstract}

\section{CCS CONCEPTS}

-Information systems $\rightarrow$ Recommender systems; • Computing methodologies $\rightarrow$ Discourse, dialogue and pragmatics.

\section{KEYWORDS}

dialogue system, recommender system, deep reinforcement learning, end-to-end memory networks

\section{ACM Reference Format:}

Daisuke Tsumita and Tomohiro Takagi. 2019. Dialogue based recommender system that flexibly mixes utterances and recommendations. In IEEE/WIC/ACM International Conference on Web Intelligence (WI '19), October 14-17, 2019, Thessaloniki, Greece. ACM, New York, NY, USA, 8 pages. https://doi.org/10. $1145 / 3350546.3352500$

\section{INTRODUCTION}

The development of goal-oriented dialogue systems that provide customer and consultation services is a research area that is growing remarkably with rising demand. An important factor in the design of dialogue systems is the definition of a dialogue strategy that determines what responses are returned to the user utterances. In conventional goal-oriented dialogue systems, a rule-based dialogue strategy that matches the user dialogue and returns an appropriate response is mainstream, but this assumes various user utterances, and defining a huge number of rules is needed. Therefore, a lot of

Permission to make digital or hard copies of all or part of this work for personal or classroom use is granted without fee provided that copies are not made or distributed for profit or commercial advantage and that copies bear this notice and the full citation on the first page. Copyrights for components of this work owned by others than ACM must be honored. Abstracting with credit is permitted. To copy otherwise, or republish, to post on servers or to redistribute to lists, requires prior specific permission and/or a fee. Request permissions from permissions@acm.org.

WI '19, October 14-17, 2019, Thessaloniki, Greece

(C) 2019 Association for Computing Machinery.

ACM ISBN 978-1-4503-6934-3/19/10 ..\$15.00

https://doi.org/10.1145/3350546.3352500

\author{
Tomohiro Takagi \\ Dept. of Computer Science, \\ Meiji University, Japan \\ takagi@cs.meiji.ac.jp
}

labor is required to add functions and modify rules. Model-based dialogue systems based on frameworks such as bandit algorithms and reinforcement learning are currently attracting attention for the problems of rule-based dialogue strategies. Moreover, in conventional goal-oriented dialogue systems, the goal to be achieved is determined, and the goal can be achieved simply by presenting the user's desired solution.

On the other hand, in situations where users themselves do not explicitly understand their needs, it is necessary to construct a recommender system for users. However, since the accuracy of the recommender system itself is not necessarily high, it is conceivable that in such situations, the recommendation results do not meet the user needs from the beginning of the dialogue. In this case, it is necessary to guide the user toward a solution that he is satisfied with while searching for potential needs by confirming and correcting the reasons for nonconformity and repeatedly presenting other recommendation results. In addition, by mixing information collection through conversations with the user and the presentation of recommendation results, it is necessary to improve the efficiency by reducing the number of conversations as a whole. It is difficult to construct such a system that naturally intermingles utterances with recommendations in a conversation as humans would do, and most of the previous research designed systems that separate normal utterances and recommendations.

In this paper, we define a recommender system using user utterance information and define a dialogue strategy based on the recommendations. Then, we propose a dialogue system with flexible behavior that mixes normal utterances and recommendations as humans would do.

\section{RELATED WORK}

Goal-oriented dialogue systems can be classified into rule-based dialogue systems and model-based dialogue systems. [3] proposed a rule-based dialogue system that supports users searching for an item through conversation. This system learns the preferences of users and succeeds in conducting efficient dialogue.

Many attempts are being made to build model-based goal-oriented dialogue systems, and in particular, research using deep learning and reinforcement learning gets a lot of attention. [8] proposed an end-to-end goal-oriented dialogue system with a neural network. However, the dialogue system [8] has the disadvantage that it requires a huge number of labels since the model is updated by supervised learning.

On the other hand, bandit algorithms and reinforcement learning are called semi-supervised learning and are frameworks that can learn better behavior without requiring labels. 


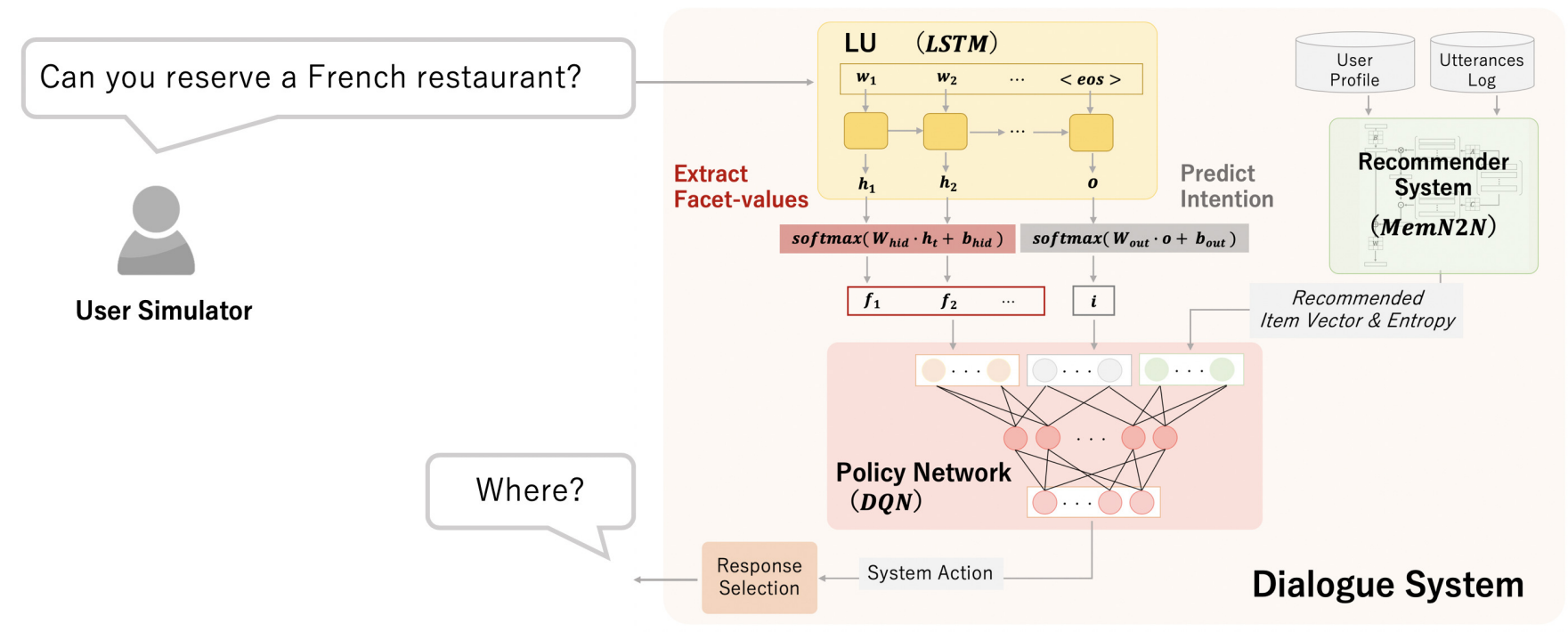

Figure 1: Overview of the proposed system

[5] proposed a conversational recommender system using Probabilistic Matrix Factorization, which uses a bandit algorithm to select response sentences. In addition, [7] and [1] constructed dialogue systems by end-to-end reinforcement learning in the dialogue manager. [11] has devised a dialogue strategy by deep reinforcement learning in the task of movie-ticket booking. [11] proposed a framework for end-to-end learning from user utterances comprehension to agent ${ }^{1}$ response generation. In their research, however, because of the nature of the task of movie-ticket booking, it is assumed that the target movie of the user has already been decided, and no recommendation by the agent is made.

There is also research that focuses on recommendation problems in dialogue. For the task of restaurant reservation, in addition to the dialogue strategy by deep reinforcement learning, [12] proposed a model that makes recommendations based on the dialogue state and the user's action history and succeeded in efficient conversations using a recommender system. However, the outputs of their recommender system are not considered in the dialogue strategy.

\section{PROPOSED FRAMEWORK}

In this research, we assume a dialogue in the situations where users search for a restaurant. The goal of our dialogue system is to reduce wasteful utterances, find out the user's desired conditions with as short a dialogue as possible, and to recommend a restaurant that the user is satisfied with.

\subsection{Overview}

In this research, based on the dialogue system developed by [11], we designed an agent that decides dialogue actions in the framework of deep reinforcement learning, and the prediction by the recommender system is used for the dialogue strategy. Figure 1

In this paper, we call the model that uses a dialogue strategy itself the agent for the whole proposed dialogue system. shows the configuration of the user simulator (left in Figure) and our proposed system (right in Figure).

The goal of our conversational recommender system is to recommend in short dialogues stores that users are satisfied with, and it is necessary to achieve the goal more efficiently while collecting information by questions. For that purpose, several factors are important, and our proposed system is roughly divided into three modules: language understanding, recommender system, policy network.

Language Understanding (LU): When an utterance is given in the form of a natural language from the user simulator that utters in a rule-based manner, the system first receives the user's utterance by LU. The LU component extracts the user's utterance intention and facet-value pairs. The facet means a category of items, and in the restaurant reservation, it corresponds to search conditions such as the restaurant genre, location, and price range. LU predicts facets included in the user's utterance and estimates what the value of the facets is.

Recommender System: This module predicts and outputs vectors of the restaurant that the user is satisfied with on basis of the user's profile information and the current conversation. We used End-to-End Memory Networks[6] as a recommender system model. In this paper, we also show the usefulness of End-to-End Memory Networks in recommendation tasks using utterance information.

Policy Network: This is a module for determining the utterance actions of the system. Based on the information of facet-values and the user's utterance intention, as well as the recommendation results and the certainty of the recommendation, the utterance for achieving efficient dialogue is estimated by deep reinforcement learning.

In this section, we describe the details of each module in our proposed system. 


\subsection{Predicting Intention and Extracting Facet-values}

As a function in a goal-oriented dialogue system that makes recommendations, it is required to estimate what kind of intention the user utters from the user's utterance. Similarly, it is necessary to extract the facet-values that the user asks for in regards to the restaurant. In this research, we use Long Short-term Memory (LSTM), which has been successful for generative models and time-series predictions, as an LU model that simultaneously estimates the user's utterance intention and the facet-values. We show the flow of prediction by LSTM below.

(1) An utterance sentence is divided into words and converted into word vectors, which are input to LSTM. The word vectors are one-hot vectors whose number of dimensions is the total vocabulary size, and it has a form in which 1 is placed at the dimensional position corresponding to the word. Let $\boldsymbol{w}_{t}$ be the $\boldsymbol{t}$-th word in the utterance sentence. Then, among the hidden expressions obtained from LSTM, let $o$ be the final output vector and $\boldsymbol{h}_{1}, \boldsymbol{h}_{2}, \ldots, \boldsymbol{h}_{n}$ be the vector of the intermediate output before vector $\boldsymbol{o}$. Also, put the end tag of the sentence (eos) in the final cell.

$$
h_{1}, h_{2}, \ldots, h_{n}, o=\operatorname{LSTM}\left(w_{1}, w_{2}, \ldots, w_{n}, e o s\right)
$$

(2) By weighting $\boldsymbol{h}_{1}, \boldsymbol{h}_{2}, \ldots, \boldsymbol{h}_{n}$ and $\boldsymbol{o}$ differently and feeding to a softmax layer, we get the vectors $f_{1}, f_{2}, \ldots, f_{n}$ and $i$. We train LU that the vector $\boldsymbol{i}$ obtained from the final output represents the user's utterance intention, and each vector $f_{t}$ obtained from the intermediate output represents facet-values of the words $\boldsymbol{w}_{t}$.

$$
\begin{aligned}
f_{t} & =\operatorname{softmax}\left(W_{\text {hid }} \cdot h_{t}+b_{\text {hid }}\right) \\
i & =\operatorname{softmax}\left(W_{\text {out }} \cdot o+b_{\text {out }}\right)
\end{aligned}
$$

We use the vectors of the user's utterance intention and facetvalues obtained from LU for input to the policy network.

\subsection{Recommender System}

Based on the user's utterances and profile information, a restaurant that matches the user's preferences is predicted.

In this research, we construct a recommender system using Endto-End Memory Networks (MemN2N), which have been proposed in the field of question answering. MemN2N is also focused on the utterance prediction of dialogue systems with accuracy higher than other deep learning models such as Recurrent Neural Networks (RNNs) and LSTM.

MemN2N, which is used in the field of question answering, stores past utterances in the memory, and when receiving a question, it derives an answer by referring to memory using the question sentence.

In our setting, we use the profile information of the user instead of the question sentence and build a system that refers to past utterances to recommend a restaurant (store). The output of the recommender system by MemN2N is a vector whose number of dimensions is the total number of stores, and each dimension contain the probability that the corresponding store will be recommended.

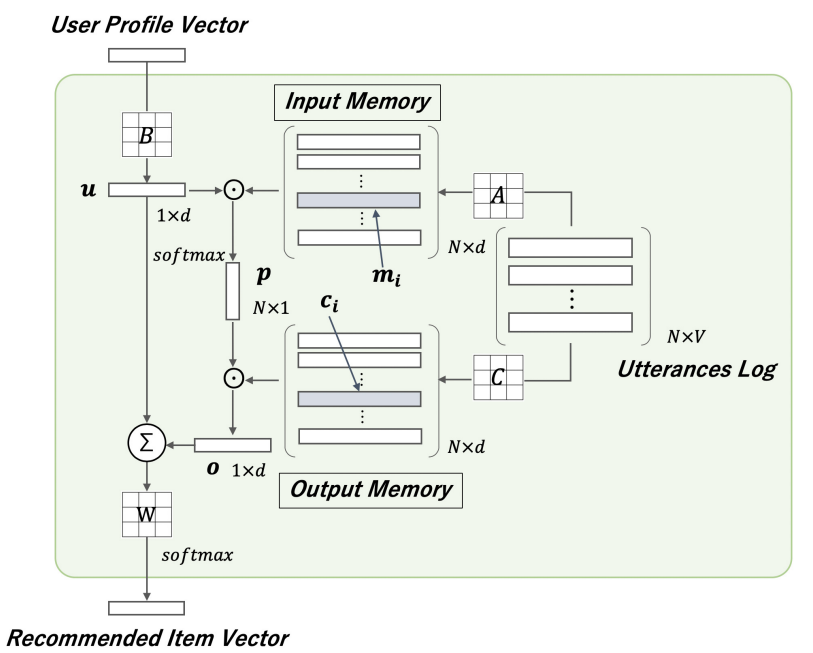

Figure 2: Single-layer version of End-to-End Memory Networks

In Figure 2, we show the structure of the recommender system based on MemN2N. What is shown here is a single-layer MemN2N, and our model has a three-layer structure.

We explain the recommendation algorithm of single-layer MemN2N. We use vectors of the user profile information described in Section 4.1 for input of the recommendation model. In addition, we prepare lists (utterances log) in which the latest $N$ utterance vectors included in the current dialogue are stored. An utterance vector has a bag-of-words representation consisting of $V$ dimensions (the vocabulary size). First, the input user profile vector is mapped by the embedding matrix $B$ to the vector $u$ of dimension $d$. Also, the $\boldsymbol{i}$-th utterance vector in the utterances $\log$ is mapped to vectors $m_{i}$ and $c_{i}$ of dimension $d$ by embedding matrix $\mathrm{A}$ and $\mathrm{C}$ and stored in the input memory and the output memory, respectively. We calculate the match between $u$ and $m_{i}$ in the input memory by taking the inner product followed by a softmax and obtain the $i$-th element $p_{i}$ of the weight vector $p$.

$$
p_{i}=\operatorname{softmax}\left(u \cdot m_{i}^{\mathrm{T}}\right)
$$

Then, by calculating the inner product of the weight vector $p$ and the output memory $c_{i}$, the $i$-th element $o_{i}$ of the output vector $o$ from memory is obtained.

$$
o_{i}=p^{\mathrm{T}} \cdot c_{i}
$$

Finally, the sum of the input user profile embedding $u$ and the output vector $o$ from memory is mapped to a store number dimension vector by passing it through weight matrix $\mathrm{W}$, and then the vector is fed to a softmax layer to obtain the final output (expressing probability to recommend each store).

By using a recommender system based on MemN2N, we expect that the whole model learns recommendations that consider important parts in utterances. Specifically, because of the structure of the model, the attention mechanism should be able to make recommendations that extract important parts in utterances. 
As far as we know, this is the first approach to use MemN2N for a recommendation task based on dialogue information. In Section 5.1, we compare and verify the recommendation performance of MemN2N in our task with a traditional deep feed-forward neural network and show the usefulness of MemN2N.

The output vector of the recommender system is also used as the input of the policy network, as well as the user's utterance intention and facet-values estimated by LU.

\subsection{Policy Network}

In this research, we build an agent that uses a dialogue strategy based on deep reinforcement learning as well as the dialogue system [11].

Reinforcement Learning: In the problem setting of reinforcement learning, an environment is defined, and states $\boldsymbol{s}_{t}$ are given to an agent operating under the environment. The agent determines the action $\boldsymbol{a}_{t}$ to be taken next on the basis of the policy $\pi$ that the agent owns. Then, the environment gives the reward $\boldsymbol{r}_{t}$ that quantifies the good or bad of the agent's action and the next state $s_{t+1}$ to the agent. The above flow is defined as one step, and it is repeated until the agent achieves the goal or until it is regarded as a failure in achieving the goal under specific conditions. This cycle is called one episode, and the agent repeats the episode to update the action values calculated by its own policy.

State: We define the state $\boldsymbol{s}_{t}$ given by the environment as the form of concatenating multiple vectors in the following equation.

$$
s_{t}=v_{f} \oplus v_{i} \oplus v_{\text {item }} \oplus e_{\text {item }}
$$

Here, $\boldsymbol{v}_{f}$ is a vector of the form where a flag stands at the corresponding position of the facet-value. $\boldsymbol{v}_{i}$ is a vector representing the intentions of the user and system utterances for the past several times. $\boldsymbol{v}_{\text {item }}$ is the recommended item (store) vector predicted by the recommender system, and $\boldsymbol{e}_{\text {item }}$ is the store's entropy calculated from the recommended store vector $\boldsymbol{v}_{\text {item }}$. The purpose of using entropy $\boldsymbol{e}_{\text {item }}$ explicitly is to incorporate the degree of certainty about the recommendation result into the dialogue strategy.

Action: The actions that the agent takes in the environment are roughly divided into three: question of facet-values, the confirmation of corrections in facet-values, and recommendation of a store. In the question of facet-values, there are as many action patterns as the number of facets (e.g., question_genre, question_location, ...). Normally, other actions are also required for conversations after recommendation completion, but since the purpose of our task is recommendation in dialogue, we will not treat unnecessary action patterns for these dialogues.

Deep Policy Network: In this research, we use Deep Q-Network (DQN) [9], which is an algorithm of deep reinforcement learning, as a policy in dialogue strategy. DQN is a method of deep reinforcement learning in which action values are function-approximated by a multi-layer neural network and can be applied to tasks where the state space is continuous and the action space is discrete. In this research, we achieve the dialogue strategy in the framework of DQN, and calls the policy expressed by the neural network the policy network. The policy network is optimized to maximize the action values of the utterance to be taken next from the user's utterance intention and the facet-values and recommended item information.

\section{EXPERIMENTAL SETUP}

We prepare an offline experimental environment using a user simulator to train and evaluate our proposed system.

\subsection{Dataset}

We use the dialogue dataset developed by [2] (Personalized Dialog dataset) to create the offline experimental environment. This dataset is created for the task of predicting the next utterance from the usersystem conversations about restaurant reservation. All records of conversations made up to the reservation completion are provided, and the user's profile information such as gender, age, dietary preference, and favorite food item is given. In addition, the restaurant information of 2,400 stores is prepared as a knowledge base, and it is possible to narrow down the restaurants by the conditions of cuisine type, location, price range, and party size, that is, the facet-values. There are 12,000 dialogue sets for training, validation, and testing, respectively.

We extract templates of user and system utterances from Personalized Dialog dataset. The utterance patterns of the user include informing the facet-values, the acceptance of the store, the demand of other stores, and denial. The utterance patterns of the system have the actions of asking about each facet-value, the confirmation of corrections in facet-values, and the recommendation of a store.

In our proposed system, we use the training data to pre-train the LU and the recommender system components. We extract the utterance intention patterns and facet-values from the user's utterance sentences contained in the training data, and they are used as labels in the LU component. In the recommender system, the utterances log and the user profile are used as features, and the stores actually accepted by the user are used as labels.

In addition, we use validation data for training of the whole dialogue system and testing data for performance verification.

\subsection{User Simulator}

In the problem setting of reinforcement learning, the environment instead of a human is required to communicate with the agent. [10] collected the dialogue data about a movie-ticket booking by crowd sourcing and created a user simulator that selected response sentences based on rules.

In this research, referring to [10], we create a user simulator using user utterances extracted from Personalized Dialog dataset and treat it as an environment in reinforcement learning.

The specifications of the user simulator are shown below. In the following, the user simulator is simply called the user.

- The user has profile information (gender, age, dietary preference and favorite food item) and the desired facet-values, and there is always a restaurant that the user accepts.

- The user's facet-values may change in the middle of a conversation.

- The user always responds cooperatively to the agent, that is, the user always answers questions about facet-values. 
- A user's utterance is defined as a state, and an agent's utterance for the state is defined as an action. An agent's response to a user's utterance is considered as one step.

- The user provides appropriate rewards for the agent's action.

- The episode terminates when the agent recommends a restaurant that the user is satisfied with. Also, the maximum step length (the number of dialogue turns) is 25 , and the episode terminates even if the number of steps reaches 25 .

\subsection{Reward Definition}

We define the reward that the environment gives to the agent's utterance actions as follows. The reward has a simple design that is customarily used in dialogue system research, which is used in [11], [4], and [12].

- A positive reward of 20 will be received for the recommendation success when the user accepts the recommendation store.

- A reward of -1 will be received by the agent at each dialogue turn. This means a penalty for utterances.

- A reward of -0.5 will be received by the agent if the agent makes non-logical utterances such as asking about the facetvalues that the agent has already heard about.

The purpose of this reward design is to make the system learn not only to make the dialogue successful but also to prevent the dialogue from being broken and to reduce the number of utterances.

These rewards are used to update parameters of the policy network.

\section{EVALUATION EXPERIMENTS}

We perform two offline experiments to evaluate the performance of our proposed system. The first is performance evaluation of the recommender system alone, and the second is performance evaluation of the entire dialogue based recommender system.

\subsection{Recommender System Evaluation}

In this research, we use MemN2N as a recommender system. The research using MemN2N in constructing a recommender system based on the user's utterance information is the first approach as far as we know. Therefore, we will evaluate the performance of this model. As a comparison method, we use a simple Deep Neural Network (DNN). DNN consists of a four-layer neural network, and it has a model structure that receives the user profile vector and utterances $\log$ as input, and it outputs the recommended store vector as well as MemN2N.

Learning curves for the recommender systems are shown in Figure 3, which represents the median of the recommendation ranking (predicted by each method) of the correct answer labels. Among 2,400 stores, it can be seen that the results of DNN converges at around 500th place, whereas MemN2N reaches a value lower than the 200th place.

Table 1 shows the median (ranking_median) and average (ranking_average) of shop rankings of the correct answer labels predicted by each method and the rate of being able to be predicted as the first place (success rate). In ranking_median, the result of DNN is 474.86, while the one of MeMN2N succeeds in the prediction at 137.54. Also, regarding ranking_average, MemN2N has a fairly

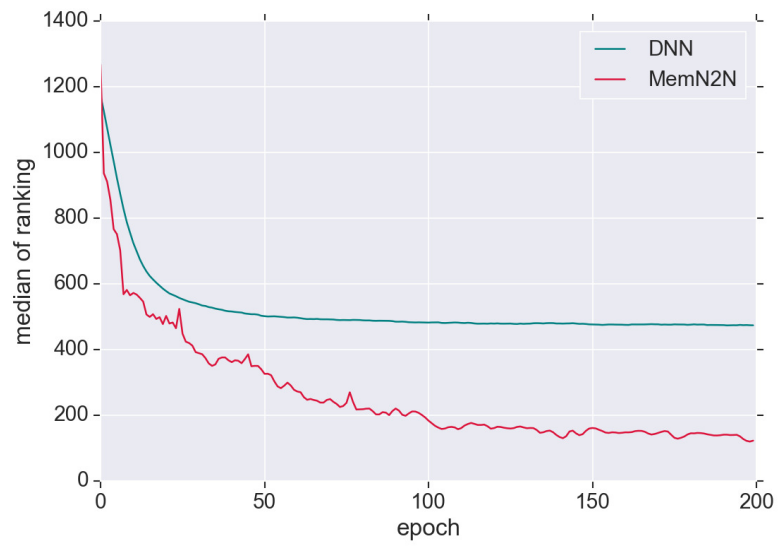

Figure 3: Median of recommendation ranking

Table 1: Performance comparison of recommender systems

\begin{tabular}{cccc}
\hline & ranking_median & ranking_average & success rate \\
\hline \hline DNN & 474.86 & 300.25 & 0.002 \\
MemN2N & $\mathbf{1 3 7 . 5 4}$ & $\mathbf{1 7 . 6 2}$ & $\mathbf{0 . 0 5 9}$ \\
\hline
\end{tabular}

good performance of 17.62. Looking at the success rate, the rate at which the correct stores were predicted to be the first place, that is, the success rate of recommendation was about 30 times as high as 0.059 for MemN2N, whereas DNN was 0.002 .

From the above, it can be said that the recommendation model using MemN2N in dialogue tasks proposed in this paper is useful.

\subsection{Dialogue System Evaluation}

We evaluate the performance of the whole dialogue based recommender system using the user simulator environment described in Section 4.2. Therefore, we prepare 12,000 pieces of profile information of users and desired facet-values for training and evaluation and design the user simulator to utter on the basis of these.

5.2.1 Evaluation Method. In this research, the shorter the number of utterances in the dialogue system, the less effort the user needs, so it can be considered as a good dialogue strategy. Also, the success rate of dialogue is defined as the success rate of recommending stores, and the dialogue is successful when the user accepts the system recommendation. Using these indicators, we compare our proposed system with a rule-based dialogue system (RuleAgent) that makes a recommendation for stores after confirming all facet-values and the dialogue system of [12] (CRM). CRM has a policy network of deep reinforcement learning and can take recommendation actions, but it differs from our system in that the recommendation results are not used for input in the policy network. We change the recommender system of CRM to MemN2N which is the same as our system, and we use CRM as a baseline method that is designed to make recommendations using current dialogue information.

In Section 5.2.2, we verify whether the certainty of the recommendation results is reflected in the dialogue strategy. 
Also, in Section 5.2.3, we compare the above two methods with our proposed system using evaluation metrics such as the number of utterances and the success rate of dialogue. In addition, we confirm the contents of conversations with the trained dialogue system and evaluate qualitatively.

5.2.2 Evaluation of the Policy Network. We verify whether the policy network takes the utterance action on the basis of the store vector outputted by the recommender system. The proposed system makes it easy to capture the degree of certainty about the recommendation results by explicitly giving the store's entropy. Here we confirm what kind of utterance action the system takes when changing the value of entropy given as input of the policy network. There are six patterns of utterance action that the dialogue system can take: asking about cuisine type, location, price range, number of people (request_cuisine, request_location, request_price,

request_number), confirming if there are any corrections in facetvalues (correction), and recommendation (recommend).

In verification, we assume a dialogue state in which all facetvalues have already been heard. Therefore, the dialogue systems should not take utterance actions to ask about facet-values and are expected to take either correction or recommend.

Table 2 shows the probability of selecting each utterance action in the trained CRM dialogue system. In CRM, since the selection rate of recommend is the highest at 0.254 , the recommendation action is always selected under this situation regardless of the prediction result of the recommender system.

In our proposed system, since the vector of the store to be recommended and the store's entropy are received at the input of the policy network, the selection rate of the utterance actions changes according to these values. When the entropy of stores is large, the difference between the element values of the store vector is small, which means that the certainty of the store to be recommended is low. On the other hand, when the entropy is small, the element values of the store vector are biased, and it can be said that the certainty of the store to be recommended is high.

Figure 4 shows the relationship between the entropy of the store and the selection rate of each utterance action calculated from the store vector predicted by the recommender system.

As shown in Figure 4, when the value of entropy is small, the action recommend is likely to be selected because high certainty of the store to be recommended is taken into consideration. As the value of entropy gets larger, the action correction tends to be selected, and conversely, recommend is less likely to be selected. From these results, it can be said that the policy network in our proposed system can select the utterance actions in consideration of the prediction results of the recommender system.

5.2.3 Results. Figure 5 shows learning curves regarding the number of utterances by the dialogue systems in one episode. ProposedSys represents the number of utterances by our proposed system for evaluation data. Also, CRM represents the number of utterances by the baseline dialogue system [12], and RuleAgent represents that by the rule-based dialogue system. While the rulebased system utters approximately 21.6 times, the trained CRM does so 19.4 times (at the right end of the CRM graph in Figure 5), modelbased dialogue systems outperformed the others. Furthermore, the
Table 2: Probability of CRM dialogue actions

\begin{tabular}{cc}
\hline actions & probability \\
\hline \hline request_cuisine & 0.087 \\
request_location & 0.120 \\
request_price & 0.203 \\
request_number & 0.124 \\
correction & 0.212 \\
recommend & 0.254 \\
\hline
\end{tabular}

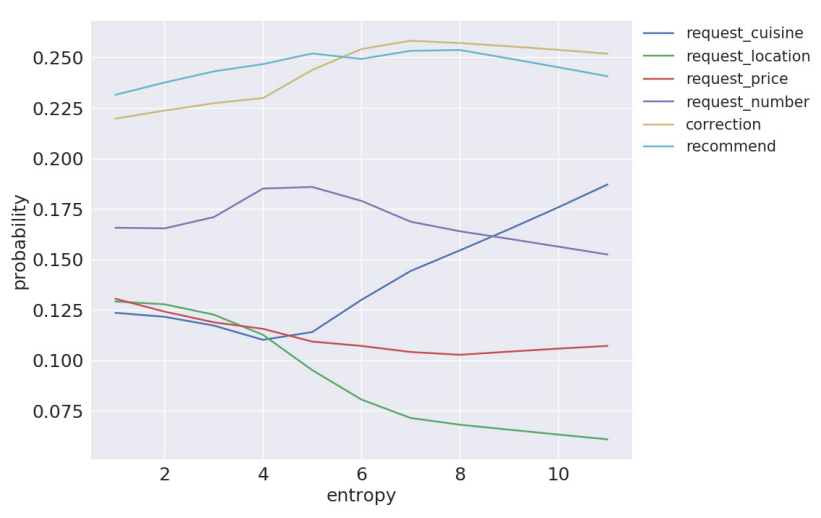

Figure 4: Probability of dialogue actions with different store entropies

trained agent of our proposed system can perform efficient conversations that exceed the baseline with approximately 16.9 times (at the right end of the ProposedSys graph in Figure 5).

The graphs shown in Figure 6 represent the success rate of dialogue under the same conditions as Figure 5. As the training of our agent is repeated, the success rate of dialogue improves, and it exceeds that of the rule-based system and CRM.

We summarize the number of utterances and the success rate of dialogue in RuleAgent, and trained models of CRM and ProposedSys in Table 3.

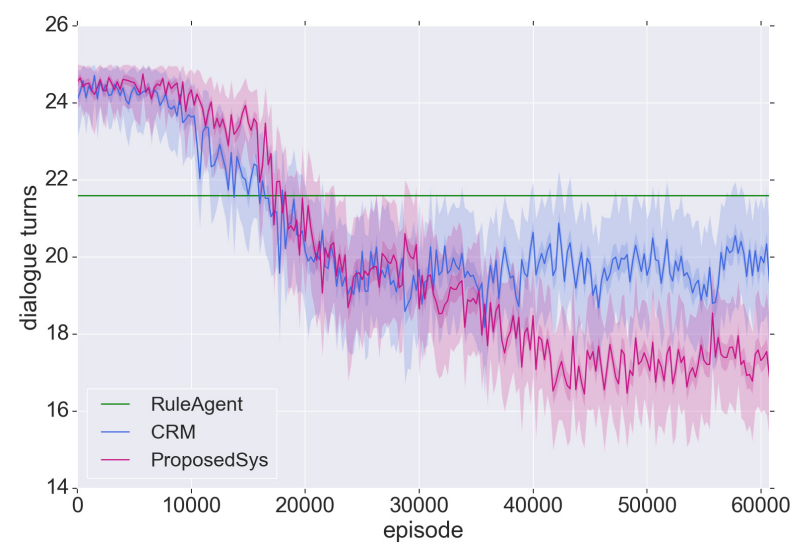

Figure 5: Number of utterances 
Table 4 shows the contents of the conversations by our trained system. Rule-based dialogue systems find all facet-values and then switch to store recommendation. However, in the dialogue of our proposed system shown on the left side of Table 4, only the necessary facet-values are heard, and the dialogue succeeds efficiently by switching correction and recommend according to the certainty of recommendation. If it does not work as well as the first recommendation, it can be seen that our system asks about the facet-values again and works to search for the condition that the user really wants.

\section{Table 3: Performance comparison of dialogue systems}

\begin{tabular}{lccc}
\hline & RuleAgent & CRM & ProposedSys \\
\hline \hline dialogue turns & 21.62 & 19.36 & $\mathbf{1 6 . 8 6}$ \\
success rate & 0.36 & 0.53 & $\mathbf{0 . 7 8}$ \\
\hline
\end{tabular}

The right side of Table 4 shows one failure dialogue example generated by our system. Although the system heard all of the facet-values from the beginning, the dialogue failed because the recommendation did not succeed in 25 utterances.

\subsection{Discussion}

In this research, we design a recommender system using user utterance information and achieve a dialogue strategy using prediction by the recommender system.

As for the recommender system using user utterances, we have shown that using MemN2N is more effective in terms of performance than a simple deep neural network model in this task.

In MemN2N, it is considered that the attention mechanism for the past user's utterance by the user profile improves the performance by enabling recommendations to capture the important parts in the utterance information. In this research, we verify only the dialogue with the user simulator, but in real problems, more important information in the recommendations should be hidden in the user's utterances. For example, if the user first requested a restaurant location, the location should be more important than the price range. Therefore, the recommendations using the user's utterance information as at this time are also useful in real problems.

In addition, our proposed system succeeded in shortening the number of utterances by the dialogue system and improved the success rate compared to the rule-based system and the baseline system CRM. As shown in Section 5.2.2, our proposed system uses the prediction result of the recommender system to determine the next utterance action, enabling utterance decision based on the certainty of recommendation. Therefore, even if the facet-values are not fully understood, if the certainty of recommendation is high, the recommendation action may be taken. It is thought that the flexible decision of utterances using the recommendation results influenced the efficiency of dialogue.

On the other hand, in qualitative evaluation, many conversations that could not satisfy the user's needs were also confirmed. In our task, since the accuracy of the recommendation results is greatly related to the success of the dialogue, the success of the dialogue becomes extremely difficult in a situation where the recommender

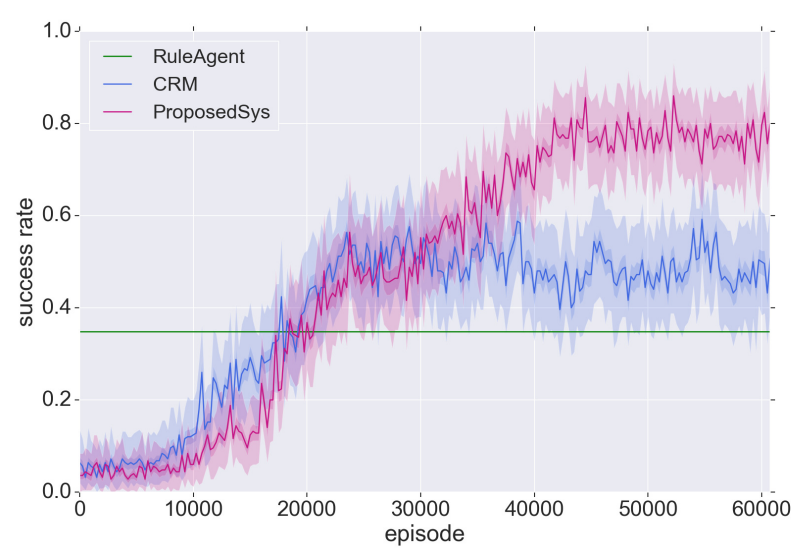

Figure 6: Success rate of dialogue

system cannot properly understand the user's needs. Also, in this research, the number of utterances in one episode is limited. As a rule-based dialogue system, therefore, even if systems use the dialogue strategy that hears all facet-values at the beginning of the dialogue and then recommends stores continuously, it is difficult to make a dialogue successful. From the above, we cannot generally conclude that a failure dialogue is due to a bad dialogue strategy in our task. In fact, as in the dialogue example on the right of Table 4, there were many failure dialogues because the recommendation results were not accepted by the user, even though all facet-values could be heard. This failure dialogue example is considered to be rather excellent as a dialogue strategy in that our agent understands the situation that the certainty of recommendation is low and the recommendation fails continuously, and it confirms again whether a correction of facet-values is necessary.

\section{CONCLUSION AND FUTURE WORK}

In this paper, we deal with goal-oriented dialogues in situations where the user's goals are not explicit, and in a problem that requires a recommendation for the user, we propose a system that uses a dialogue strategy based on the prediction of a recommender system using utterance information. Furthermore, we achieve a dialogue system that enables human-like and efficient conversations, such as the combination of collecting facet-values and recommendations and flexibly uttering according to the situation.

On the other hand, there are cases where the system cannot succeed in the dialogues because of the accuracy of recommendations, and it is considered that the accuracy of recommendations using only the user's utterance information and profile information is limited. In the future, it is necessary to consider improving the performance of the recommender system by combining it with other methods based on user's long-term preferences using purchase history.

Also, in this research, we verify only with very limited utterance behaviors of users and the system and build a dialogue system under the limited problem setting of restaurant reservation. In future research, it is necessary to construct a dialogue system that assumes not only a single task such as restaurant reservation but 
Table 4: Sample dialogues generated by the proposed system with user simulator

\begin{tabular}{|c|c|}
\hline success dialogue example & failure dialogue example \\
\hline $\begin{array}{l}\text { User: Can you book a table in Madrid? } \\
\text { Agent: What type of cuisine would you like to eat? } \\
\text { User: With Italian food. } \\
\text { Agent: May I suggest this option: } \\
\text { resto_madrid_cheap_italian_7stars_2 } \\
\text { User: No I don't like that. } \\
\text { Agent: Is there any change? } \\
\text { User: Instead could it be with British cuisine. } \\
\text { Agent: Is there any change? } \\
\text { User: No. } \\
\text { Agent: May I suggest this option: } \\
\text { resto_madrid_moderate_british_7stars_1 } \\
\text { User: Let's do it. }\end{array}$ & 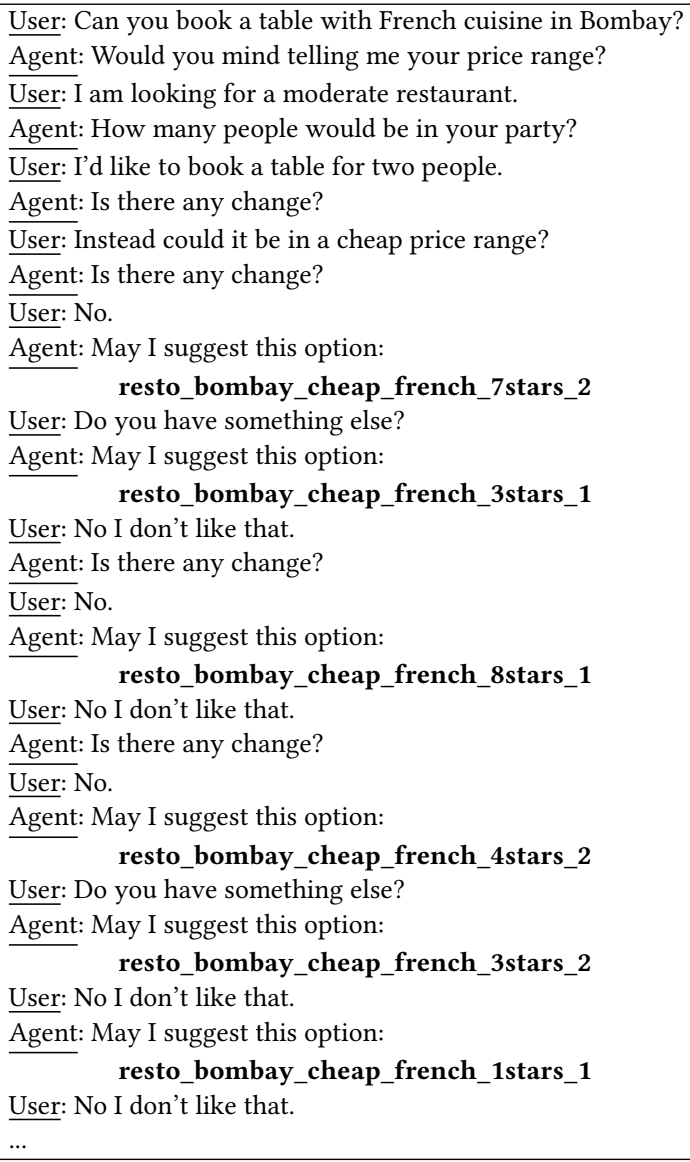 \\
\hline $\begin{array}{l}\text { The agent hears only the necessary facet-values and } \\
\text { succeeds in conducting efficient conversations. }\end{array}$ & $\begin{array}{l}\text { The agent fails in recommendation many times, and } \\
\text { finally the episode terminates without a successful } \\
\text { dialogue. }\end{array}$ \\
\hline
\end{tabular}

also tasks involving open domain dialogues and various utterance actions.

\section{REFERENCES}

[1] Xiujun Li Jianfeng Gao Yun-Nung Chen Faisal Ahmed Li Deng Bhuwan Dhingra, Lihong Li. 2017. Towards End-to-End Reinforcement Learning of Dialogue Agents for Information Access. In Proceedings of the 55th Annual Meeting of the Association for Computational Linguistics.

[2] Boi Faltings Chaitanya K. Joshi, Fei Mi. 2017. Personalization in Goal-oriented Dialog. In NIPS '17.

[3] Pat Langley Cynthia A Thompson, Mehmet H Goker. 2004. A Personalized System for Conversational Recommendations. In fournal of Artificial Intelligence Research 21.

[4] Shuangyin Li Jiajun Li Qiang Yang Kaixiang Mo, Yu Zhang. 2018. Personalizing a Dialogue System with Transfer Reinforcement Learning. In Association for the Advancement of Artificial Intelligence '18.

[5] Katja Hofmann Konstantina Christakopoulou, Filip Radlinski. 2016. Towards Conversational Recommender Systems. In KDD ' 16.

[6] Jason Weston Rob Fergus Sainbayar Sukhbaatar, Arthur Szlam. 2015. End-To-End Memory Networks. In NIPS '15.

[7] Maxine Eskenazi Tiancheng Zhao. 2016. Towards End-to-End Learning for Dialog State Tracking and Management using Deep Reinforcement Learning. In Proceedings of the SIGDIAL '16.
[8] Nikola Mrksic Lina M Rojas-Barahona Pei-Hao Su Stefan Ultes David Vandyke Steve Young Tsung-Hsien Wen, Milica Gasic. 2016. A Network-based End-to-End Trainable Task-oriented Dialogue System. In Proceedings of the 15th Conference of the European Chapter of the Association for Computational Linguistics: Volume 1, Long Papers.

[9] David Silver Alex Graves Ioannis Antonoglou-DaanWierstra Martin Riedmiller Volodymyr Mnih, Koray Kavukcuoglu. 2013. Playing Atari with DeepReinforcement Learning. In NIPS Deep Learning Work-shop '13.

[10] Bhuwan Dhingra Lihong Li Jianfeng Gao Yun-Nung Chen Xiujun Li, Zachary C. Lipton. 2016. A User Simulator for Task-Completion Dialogues.

[11] Lihong Li Jianfeng Gao Asli Celikyilmaz Xiujun Li, Yun-Nung Chen. 2017. TaskCompletion Neural Dialogue Systems. In Proceedings of the The 8th International foint Conference on Natural Language Processing (IFCNLP '17).

[12] Yi Zhang Yueming Sun. 2018. Conversational Recommender System. In SIGIR 